\title{
Ethanol extract of baked Gardeniae Fructus exhibits in vitro and in vivo anti-metastatic and anti-angiogenic activities in malignant cancer cells: Role of suppression

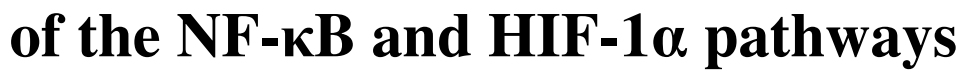

\author{
MINJU IM ${ }^{*}$, AEYUNG KIM ${ }^{*}$ and JIN YEUL MA \\ Korean Medicine (KM) Application Center, Korea Institute of Oriental Medicine (KIOM), \\ Dong-gu, Daegu 701-300, Republic of Korea
}

Received July 28, 2016; Accepted September 26, 2016

DOI: $10.3892 /$ ijo.2016.3742

\begin{abstract}
Gardeniae Fructus (GF, Zhi $\mathrm{Zi}$ in China), a fruit of Gardenia jasminoides Ellis, has been used in traditional medicine to reduce inflammation and headache and to treat hepatic disorders, hypertension, and icterus. In recent studies, extract of raw or stir-baked GF was shown to have pharmacological activities for viral infection, thrombosis, hyperlipidemia, convulsion, inflammation, oxidative stress, and others. In addition, baked GF extract suppressed the proteolytic activities and altered the cellular morphology of tumor cells. However, the effects of ethanol extract of baked GF (EBGF) on the metastatic and angiogenic capacities of malignant tumor cells and its detailed mechanism of action have not been reported. In this study, we found that EBGF significantly inhibited phorbol 12-myristate 13-acetate (PMA)-induced MMP-9 and -13 and UPA expression via suppression of PMA-induced nuclear translocation of $\mathrm{NF}-\kappa \mathrm{Bp} 65$. Metastatic potential, including migration, invasion, and colonization, was substantially reduced by EBGF with no cytotoxicity. In addition, EBGF attenuated tumor-induced angiogenesis, including microvessel sprouting, migration of endothelial cells (ECs), and tube formation of ECs, by inhibiting the release of pro-angiogenic factors from tumor cells. In C57BL/6 mice, we observed that daily administration of $\mathrm{EBGF}$ at 50 and $100 \mathrm{mg} / \mathrm{kg}$ suppressed metastatic colonization of B16F10 melanoma cells in the lungs. Furthermore, EBGF administration did not cause adverse effects, suggesting that EBGF is safe and may be a potential herbal medicine capable of controlling metastatic malignant cancers.
\end{abstract}

Correspondence to: Dr Jin Yeul Ma, Korean Medicine (KM) Application Center, Korea Institute of Oriental Medicine (KIOM), 70 Chumdan-ro, Dong-gu, Daegu 701-300, Republic of Korea E-mail: jyma@kiom.re.kr

*Contributed equally

Key words: cancer, metastasis, angiogenesis, matrix metalloproteinases

\section{Introduction}

During cancer progression, tumor cells actively interact with the tumor microenvironment, including extracellular matrices (ECMs), cytokines, growth factors, and neighboring cells, such as endothelial cells, macrophages, fibroblasts, and neutrophils, through which they acquire the capacity for migration, invasion, metastasis, and angiogenesis (1). Metastatic spread of tumors after surgical excision increases the risk of mortality in cancer patients and remains a major obstacle for the complete treatment of malignant tumors. During metastasis, tumor cells undergo a multistep process known as the metastatic cascade, which includes disconnection from ECMs, degradation of the surrounding tissues, entrance into the circulation, migration and arrival at secondary sites, extravasation, and establishment of secondary metastatic foci $(2,3)$. Several regulatory factors are overexpressed in tumors as well as the surrounding stromal cells and promote the metastatic process. Among them, matrix metalloproteinases (MMPs), zinc-dependent endopeptidases, are regarded as the most important effectors, and they collectively degrade diverse substrates in the extracellular milieu (4-6). Based on their specificity for substrates, MMPs can be classified as gelatinases, collagenases, stromelysins, and membrane-type MMPs. Elevated expression and activity of MMPs in primary tumors and/or plasma are positively correlated with rapid progression and high incidence of metastasis in diverse types of cancer as well as shorter survival time. In addition to their proteolytic activities, MMPs contribute to tumor cell proliferation by modulating the bioavailability of growth factors and to the formation of tumor-associated vasculature, which promotes tumor dissemination, by degrading the vascular basement membrane and remodeling ECMs $(7,8)$. Therefore, MMPs are considered valuable diagnostic markers as well as potential therapeutic targets for managing malignant tumors.

Angiogenesis plays pivotal roles in the development of malignant tumors at multiple levels and is controlled by the balance of endogenous stimulators and inhibitors that are secreted by tumor cells and host cells, including endothelial cells, fibroblasts, stromal cells, and immune cells, in response 
to tumor cells $(9,10)$. Initially, tumors are avascular masses that depend on the pre-existing vasculature in their microenvironment. However, when tumor cells grow beyond the extent of passive diffusion for oxygen and nutrient, they become hypoxic. Hypoxia causes an imbalance between the production of pro- and anti-angiogenic factors, which is known as the angiogenic switch, via overexpression and stabilization of hypoxia-inducible factor (HIF)-1 $\alpha$, leading to enhancement of blood vessel formation (11-13). Newly formed blood vessels within hypoxic tumors act as a path to supply sufficient oxygen and nutrients, eliminate waste products, and migrate and infiltrate to the circulation, thus permitting rapid growth and metastasis. Therefore, targeting tumor-induced angiogenesis has been an important strategy for cancer therapy.

Gardenia jasminoides Ellis (GJE, family Rubiaceae) has long been used in Asian countries as a traditional herbal medicine for the treatment of inflammation, hepatic diseases, hypertension, edema, jaundice, and headache $(14,15)$. The ripe fruit of GJE, Gardeniae Fructus (GF, Chinese name, $\mathrm{Zhi} \mathrm{Zi}$ ), has been used in traditional medicine and has various pharmacological effects, such as anti-oxidant, antiinflammatory, anti-thrombotic, anti-hyperlipidemic, anti-viral, anti-convulsive, anti-hyperuricemic, neuroprotective, and anxiolytic activities (16-20). In addition, stir-baked GF extract was reported to inhibit MMP-16 activity and alter the cellular morphology of HT1080 human fibrosarcoma cells with no cytotoxicity (21). However, studies on the anti-metastatic and anti-angiogenic effects of baked GF and its underlying mechanisms have not been performed.

In this study, we aimed to determine whether ethanol extract of baked GF (EBGF) can control malignant tumor cells by inhibition of metastasis and angiogenesis using in vitro assays, ex vivo rat aortic ring assays, and an in vivo pulmonary metastasis model. Furthermore, we elucidated the underlying anti-metastatic and anti-angiogenic activities of EBGF in detail.

\section{Materials and methods}

Preparation of EBGF. Baked G. Fructus was purchased from Yeongcheon Oriental Herbal Market (Yeongcheon, Korea) and stored in the herbal bank of the Korean Medicine (KM) Application Center. To prepare EBGF, $30 \mathrm{~g}$ of baked G. Fructus was crushed to a powder, soaked in $300 \mathrm{ml}$ of $70 \%$ ethanol, and then extracted by shaking at $100 \mathrm{rpm}$ for $24 \mathrm{~h}$ at $40^{\circ} \mathrm{C}$. After filtering the extract using a testing sieve $(150 \mu \mathrm{m}$, Retsch Haan, Germany), the sample was lyophilized and then stored in a desiccator at $-20^{\circ} \mathrm{C}$. The total collected EBGF was $9.11 \mathrm{~g}$, and the yield was $30.36 \%$.

Cells. HT1080 cells (human fibrosarcoma) and B16F10 cells (murine melanoma) were obtained from the Korean Cell Line Bank (KCLB, Seoul, Korea) and maintained in RPMI-1640 or DMEM supplemented with 10\% FBS (Biotechnics Research Inc., Lake Forest, CA, USA) and $100 \mathrm{U} / \mathrm{ml}$ penicillin $/ 100 \mu \mathrm{g} / \mathrm{ml}$ streptomycin (Cellgro, Manassas, VA, USA) in $5 \% \mathrm{CO}_{2}$ at $37^{\circ} \mathrm{C}$. Human umbilical vein endothelial cells (HUVECs) obtained from Innopharmascreen (Asan, Korea) were maintained in endothelial cell growth medium-2 (EGM-2; Promocell, Heidelberg, Germany) and used at passage 3-8.
Cell viability and colony formation assays. After cells $\left(5 \times 10^{3} /\right.$ well/96-well plates) were treated with or without the specified concentrations of EBGF for $48 \mathrm{~h}$, cell viability was assessed using the Cell Counting Kit-8 (CCK-8; Dojindo Laboratories, Kumamoto, Japan). To evaluate colony formation, cells $\left(5 \times 10^{2} /\right.$ well/12-well plates) were incubated in the presence or absence of the indicated concentrations of EBGF. After 7 days, colonies were stained with $0.2 \%$ crystal violet $/ 20 \%$ methanol (w/v) solution for $30 \mathrm{~min}$, and the visible colonies were counted.

Determination of MMP-9, MMP-13, and UPA mRNA levels. Total RNA from each sample was isolated using an RNA extraction solution (BioAssay Co., Daejeon, Korea) and reverse transcribed to cDNA using a 1st Strand cDNA synthesis kit (BioAssay Co.) according to the manufacturer's protocol. cDNA aliquots were amplified by polymerase chain reaction (PCR) using the following primers: hMMP-9, 5'-TCTTCCCTG GAGACTGAGAA-3' and 5'-GGCAAGTCTTCCGAGTAG TTT-3', hMMP-13, 5'-GGCAAACTTGACGATAACACC-3' and 5'-GCCCATCAAATGGGTAGAAGT-3', huPA, 5'-AGG GCAGCACTGTGAAATAGA-3' and 5'-TCTTGGACAAGC AGCTTTAGG-3', $\beta$-actin, 5'-ATGAAGATCCTGACCGAG CGT-3' and 5'-AACGCAGCTCAGTAACAGTCCG-3'.

Preparation of conditioned medium (CM). Cells were treated with the indicated concentrations of EBGF in complete media for $24 \mathrm{~h}$, washed twice with $0.5 \%$ FBS media, and then incubated for $24 \mathrm{~h}$ in $0.5 \% \mathrm{FBS}$ media. Culture media were harvested and centrifuged at $12,000 \mathrm{rpm}$ for $15 \mathrm{~min}$ at $4^{\circ} \mathrm{C}$ to remove cell debris, and the supernatants were collected as the CM.

MMP and UPA activity assays. MMP and UPA activity in the CM of HT1080 cells was determined using MMP Activity assay kit (Fluorometric-Green, cat. no. ab112146, Abcam, Cambridge, MA, USA) and uPA Activity assay kit (Colorimetric, cat. no. ECM600, Chemicon, Billerica, MA, USA), respectively, according to the manufacturer's protocol. In brief, for MMP activity assays, $25 \mu \mathrm{l}$ of CM was incubated with an equal volume of 2 mM APMA (4-aminophenylmercuric acid) working solution for $15 \mathrm{~min}$ and was then mixed with $50 \mu \mathrm{l}$ of the broad spectrum MMP fluorogenic peptide substrate solution in 96-well black plates (SPL, Kyounggi-do, Korea). After 1, 2 and $3 \mathrm{~h}$, green fluorescence intensity was measured in a SpectraMaxi3 microplate reader (Molecular Devices, Sunnyvale, CA, USA) set at Ex/Em 490/525 nm. For uPA activity assays, $160 \mu \mathrm{l}$ of diluted CM was mixed with $20 \mu \mathrm{l}$ of assay buffer and subsequently incubated with $20 \mu \mathrm{l}$ of the chromogenic substrate in 96 -well plates at $37^{\circ} \mathrm{C}$ for $30 \mathrm{~min}$ to $24 \mathrm{~h}$. The optical density was read at $405 \mathrm{~nm}$ in a SpectraMaxi3 microplate reader, and the relative activity was calculated from a standard curve.

Assessment of migration and invasion activities. The migration ability of the cells was assessed by a wound migration assay and Transwell migration assay as described previously (22). For invasion assays, Transwell chambers coated with $30 \mu 1$ of diluted Matrigel (BD Biosciences, Bedford, MA, USA) as the intervening barrier were used. 
Western blot analyses. For the extraction of whole cell lysates and nuclear/cytosolic fractions, M-PER Mammalian Protein Extraction reagent and NE-PER Nuclear and Cytosolic Extraction reagent (Thermo Scientific, Rockford, IL, USA) were used, respectively. Western blotting was performed as described previously (23), and proteins were visualized with a Bio-Rad Clarity ${ }^{\mathrm{TM}}$ Western ECL Substrate and ChemiDoc ${ }^{\mathrm{TM}}$ Touch Imaging System (Bio-Rad, Hercules, CA, USA). Antibodies against $\mathrm{p}-\mathrm{I} \kappa \mathrm{B} \alpha, \mathrm{t}-\mathrm{I} \kappa \mathrm{B} \alpha, \mathrm{NF}-\kappa \mathrm{Bp} 65$, p-Akt, t-Akt, p-mTOR, tubulin, and TBP were obtained from Cell Signaling Technology (Danvers, MA, USA), and an anti-HIF-1 $\alpha$ antibody was obtained from BD Biosciences.

Immunocytochemistry. After cells were seeded in 35-mm glass bottom dishes (SPL Lifesciences, Korea), they were treated with EBGF for $12 \mathrm{~h}$ and then stimulated with PMA $(5 \mathrm{nM})$ for $30 \mathrm{~min}$ or incubated under hypoxic conditions $\left(1 \% \mathrm{O}_{2}\right)$ for $6 \mathrm{~h}$ for the nuclear translocation $\mathrm{NF}-\kappa \mathrm{Bp} 65$ or HIF-1 $\alpha$ analysis, respectively. After washing with cold PBS three times, cells were subjected to immunocytochemistry as previously described (24). Alexa 488- or Alexa 568-conjugated goat anti-mouse IgG was used as a secondary antibody. After counterstaining with DAPI, cells were analyzed under a fluorescence microscope (Nikon Eclipse Ti).

Microvessel sprouting assays. Aortic rings were prepared from rat dorsal thoracic aortas as previously described (25). Rat aortic rings were cultured on Matrigel with EGM-2 for 3 days to initiate vessel outgrowth and were then further incubated with EBGF-treated or untreated HT1080 CM for 5 days. Microvessel sprouting was observed daily and imaged using a phase-contrast inverted microscope.

Tube formation assays. Endothelial cell capillary-like tube formation was assessed using a Cultrex in vitro angiogenesis assay kit (Trevigen, Gaithersburg, MD, USA) according to the manufacturer's protocol. In brief, basement membrane extract (BME) was coated on pre-chilled 96-well culture plates (50 $\mu \mathrm{l} /$ well) and polymerized for $30 \mathrm{~min}$ at $37^{\circ} \mathrm{C}$. HUVECs $\left(5 \times 10^{4}\right)$ suspended in $100 \mu \mathrm{l}$ EBGF-treated or untreated HT1080 CM were added to each well and incubated for 4-12 h at $37^{\circ} \mathrm{C}$. Tube formation was visualized under a phase contrast inverted microscope.

Proteome profiler antibody arrays. The expression profile of 55 angiogenesis/invasion-related proteins in the EBGF-treated or untreated CM was determined using a Proteome Profiler ${ }^{\mathrm{TM}}$ Human Angiogenesis Array kit (R\&D Systems, Minneapolis, MN, USA) according to the manufacturer's protocol. Blots were visualized using Bio-Rad Clarity Western ECL substrate and a ChemiDoc Touch Imaging system. For preparation of CM, HT1080 cells were incubated with or without EBGF $(500 \mu \mathrm{g} / \mathrm{ml})$ for $24 \mathrm{~h}$ in complete medium, washed twice with $0.5 \%$ FBS medium, and then incubated under hypoxic conditions $\left(1 \% \mathrm{O}_{2}\right)$ for an additional $24 \mathrm{~h}$ in $0.5 \%$ FBS medium.

In vivo pulmonary metastasis experiment. Pulmonary metastasis in C57BL/6 J mice was induced by intravenous injection of B16F10 cells ( $2 \times 10^{5}$ cells/200 $\mu \mathrm{l}$ of PBS) via the tail vein. After injection, mice were randomly divided into
3 groups ( $\mathrm{n}=5$ per group) and administered saline or EBGF daily at 50 and $100 \mathrm{mg} / \mathrm{kg}$ during the experiment. After the mice were sacrificed, the lungs were removed and weighed. After fixation with Bouin's solution (Sigma), metastatic black colonies on the lung surface were macroscopically counted. All animal experiments were approved by the Animal Care and Use Committee of the Korea Institute of Oriental Medicine (KIOM, Daejeon, Korea; reference nos. \#14-054 and \#16-003) and were performed in accordance with their guidelines.

Statistical analyses. Data are expressed as the mean \pm standard deviation (SD). Statistical significance was assessed using Student's t-test and one-way ANOVA with GraphPad Prism software (GraphPad Prism Software Inc., CA, USA). A p-value $<0.05$ was considered to be statistically significant.

\section{Results}

EBGF at non-cytotoxic concentrations reduces MMP and uPA activities in HT1080 cells. Prior to the assessment of antimetastatic activity of EBGF, we first examined its cytotoxicity in HT1080 cells using a CCK assay. As shown in Fig. 1A, EBGF at concentrations up to $500 \mu \mathrm{g} / \mathrm{ml}$ had no cytotoxic effects in the presence or absence of FBS; thus, we treated HT1080 cells with EBGF at the maximum concentration of $500 \mu \mathrm{g} / \mathrm{ml}$ in all subsequent experiments. Because the expression and activation of MMPs and UPA play central roles in promoting cancer metastasis, we initially examined the effect of EBGF on the transcriptional levels of MMPs and uPA by RT-PCR. Under resting conditions, HT1080 cells expressed low levels of MMP-9, MMP-13, and uPA, but PMA stimulation strongly elevated their expression. In contrast, EBGF treatment almost completely blocked the PMA-induced increase in the levels of MMP-9, MMP-13, and UPA (Fig. 1B). Analysis of proteolytic activities using EBGF-treated or untreated CM revealed that EBGF dramatically inhibited the MMP and UPA activities in HT1080 cells in a dose-dependent manner (MMP activity; $\mathrm{F}=76.95, \mathrm{p}<0.0001$, uPA activity; $\mathrm{F}=167.2, \mathrm{p}<0.0001$, one-way ANOVA) (Fig. 1C and D).

EBGF suppresses metastasis of HT1080 cells. To investigate the effects of EBGF on migration and invasion in vitro, we first assessed the ability of the cells to migrate across a wound region. Untreated HT1080 control cells migrated successfully, leading to 33.7 and $94.2 \%$ healing at 24 and $48 \mathrm{~h}$, respectively. However, EBGF treatment at 250 and $500 \mu \mathrm{g} / \mathrm{ml}$ for $48 \mathrm{~h}$ significantly inhibited wound migration to $\sim 65.1$ and $36.9 \%$ of that of the control cells, respectively $(24 \mathrm{~h} ; \mathrm{F}=10.2, \mathrm{p}=0.0026$, 48 h; F=212.7, p<0.0001, one-way ANOVA) (Fig. 2A). In a Transwell assay, EBGF markedly inhibited serum-induced migration and invasion compared to that of the control cells, showing reductions of $\sim 70$ and $95 \%$ at $500 \mu \mathrm{g} / \mathrm{ml}$, respectively (migration; $\mathrm{F}=170.1, \mathrm{p}<0.0001$, invasion; $\mathrm{F}=996.9, \mathrm{p}<0.0001$, one-way ANOVA) (Fig. 2B). In addition, the colony formation ability was significantly reduced by EBGF treatment in a dose-dependent manner $(\mathrm{F}=260.7, \mathrm{p}<0.0001$, one-way ANOVA) (Fig. 2C). These results indicate that EBGF exhibits potent anti-metastatic activity via suppression of MMP and uPA activities. 

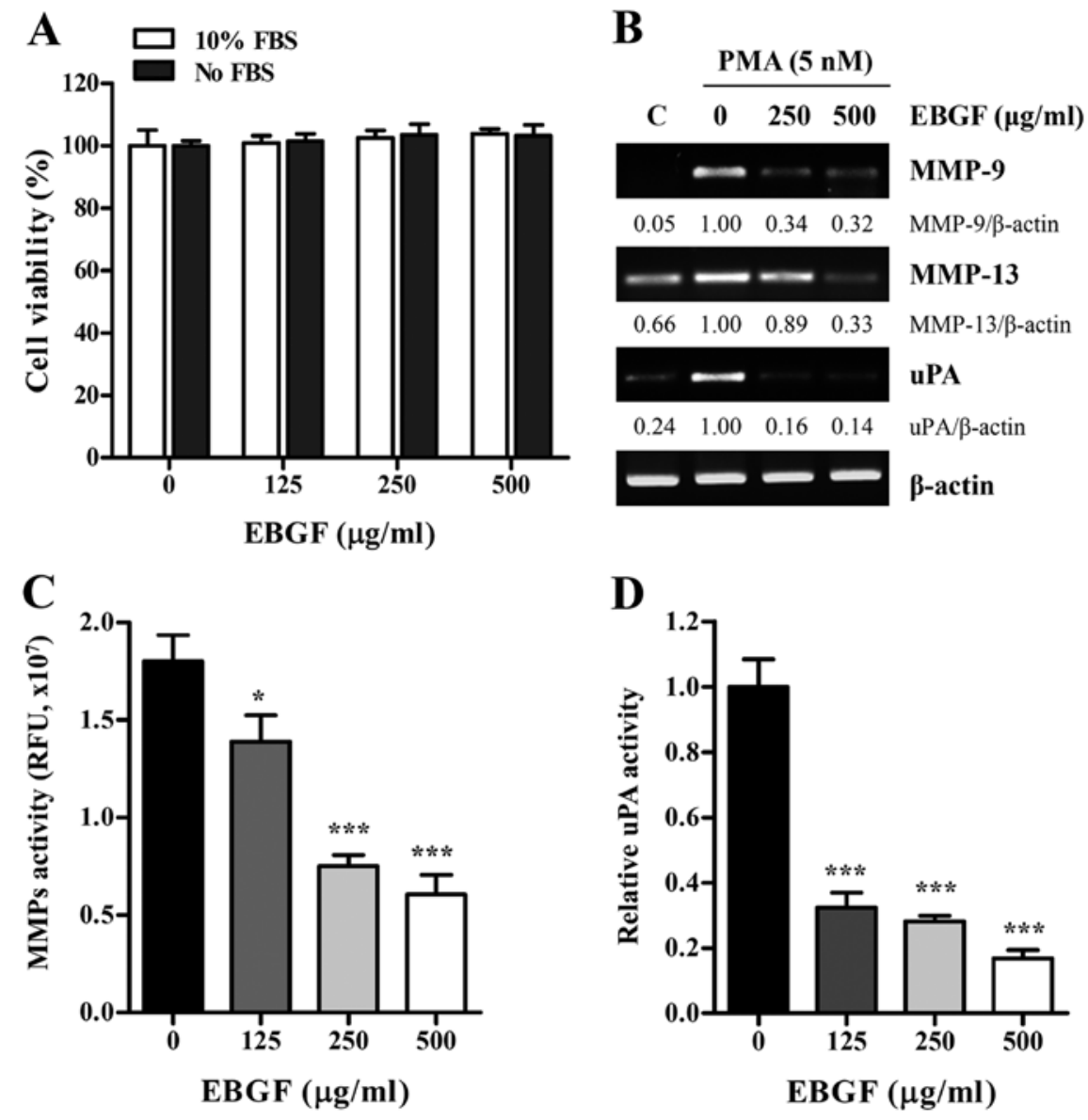

Figure 1. EBGF suppresses the MMP and uPA activities of HT1080 cells with no cytotoxicity. (A) After treating HT1080 cells with the indicated concentrations of EBGF with or without serum for $48 \mathrm{~h}$, cell viability was determined by CCK assays and is expressed as the mean \pm SD ( $\mathrm{n}=3$ per group). (B) HT1080 cells were pre-treated with 250 and $500 \mu \mathrm{g} / \mathrm{ml}$ EBGF for $12 \mathrm{~h}$ and then stimulated with PMA $(5 \mathrm{nM})$ for $24 \mathrm{~h}$ in serum-free conditions. The mRNA levels of MMP-9, MMP-13, and uPA were assessed by RT-PCR, and relative expression was determined after normalization to $\beta$-actin. (C) MMP activity in the collected CM was determined using green fluorescence substrate. Fluorescence intensity was monitored at Ex/Em 490/525 nm. (D) uPA activity in CM was measured using a specific chromogenic substrate. Data are representative of three independent experiments and are expressed as the mean \pm SD ( $n=3$ per group). Statistical significance was determined using Student's t-test. ${ }^{*}<<0.01$ and ${ }^{* * *} \mathrm{p}<0.001$ vs. untreated control.

EBGF blocks PMA-induced $N F-\kappa B$ activation in HT1080 cells. To further elucidate the mechanisms by which EBGF suppresses metastatic potential, we examined whether EBFG prevents the activation of the transcription factor NF- $\kappa B$, which is involved in the regulation of several proteolytic enzymes and invasion of cancer cells. NF- $\kappa \mathrm{B}$ activation occurs via translocation of the Rel family from the cytosol to the nucleus, preceded by the phosphorylation and degradation of $\mathrm{I} \kappa \mathrm{B} \alpha$ by IкB kinase. As shown in Fig. 3A, using western blotting, we confirmed that PMA stimulation increased phosphorylation and degradation of I $\mathrm{K} \mathrm{B} \alpha$, accompanied by increases in the nuclear protein level of NF- $\mathrm{kB}$ subunit $\mathrm{p} 65$. However, the PMA-induced increase in the $\mathrm{p}-\mathrm{I} \kappa \mathrm{B} \alpha / \mathrm{I} \kappa \mathrm{B} \alpha$ ratio as well as $\mathrm{p} 65$ nuclear translocation was significantly decreased in EBGFtreated HT1080 cells compared with that of control cells, indicating that PMA-induced NF- $\kappa B$ activation was efficiently blocked by EBGF. Similarly, immunocytochemistry analysis showed that EBGF substantially inhibited PMA-induced p65 nuclear translocation by approximately $83.8 \%$ compared with that of the untreated control cells $(\mathrm{F}=449.1, \mathrm{p}<0.0001$, one-way ANOVA) (Fig. 3B). Taken together, these results suggest that EBGF inhibits the metastatic potential of HT1080 cells by reducing MMP and uPA activities via suppression of NF- $\kappa \mathrm{B}$ activation.

EBGF-treated CM of HT1080 cells suppresses angiogenesis in vitro and ex vivo. In the tumor microenvironment, tumor cells produce several pro-angiogenic factors in response to stimuli such as hypoxia and inflammation and promote angiogenesis, which is essential for sustained growth and metastasis. Consistent with previous studies, we confirmed that CM from HT1080 triggered ex vivo microvessel sprouting from rat aortic rings and induced robust tube formation as well as migration of endothelial cells in vitro (Fig. 4). EBGF-treated CM had strong inhibitory effects on microvessel formation around aortic rings in a dose-dependent manner, leading to $\sim 80 \%$ inhibition at $500 \mu \mathrm{g} / \mathrm{ml}$ compared with that of the control CM ( $\mathrm{F}=331.4, \mathrm{p}<0.0001$, one-way ANOVA) (Fig. 4A). In addition, EBGF-treated $\mathrm{CM}$ did not induce a complete tube-like network of HUVECs and resulted in lower tube formation than that of the control CM ( $\mathrm{F}=281.9, \mathrm{p}<0.0001$, one-way ANOVA) (Fig. 4B). The migration of HUVECs across the Transwell with EBGF-treated CM showed reductions of $\sim 40$ and $75 \%$ at 250 and $500 \mu \mathrm{g} / \mathrm{ml}$ compared with that of the control CM, 

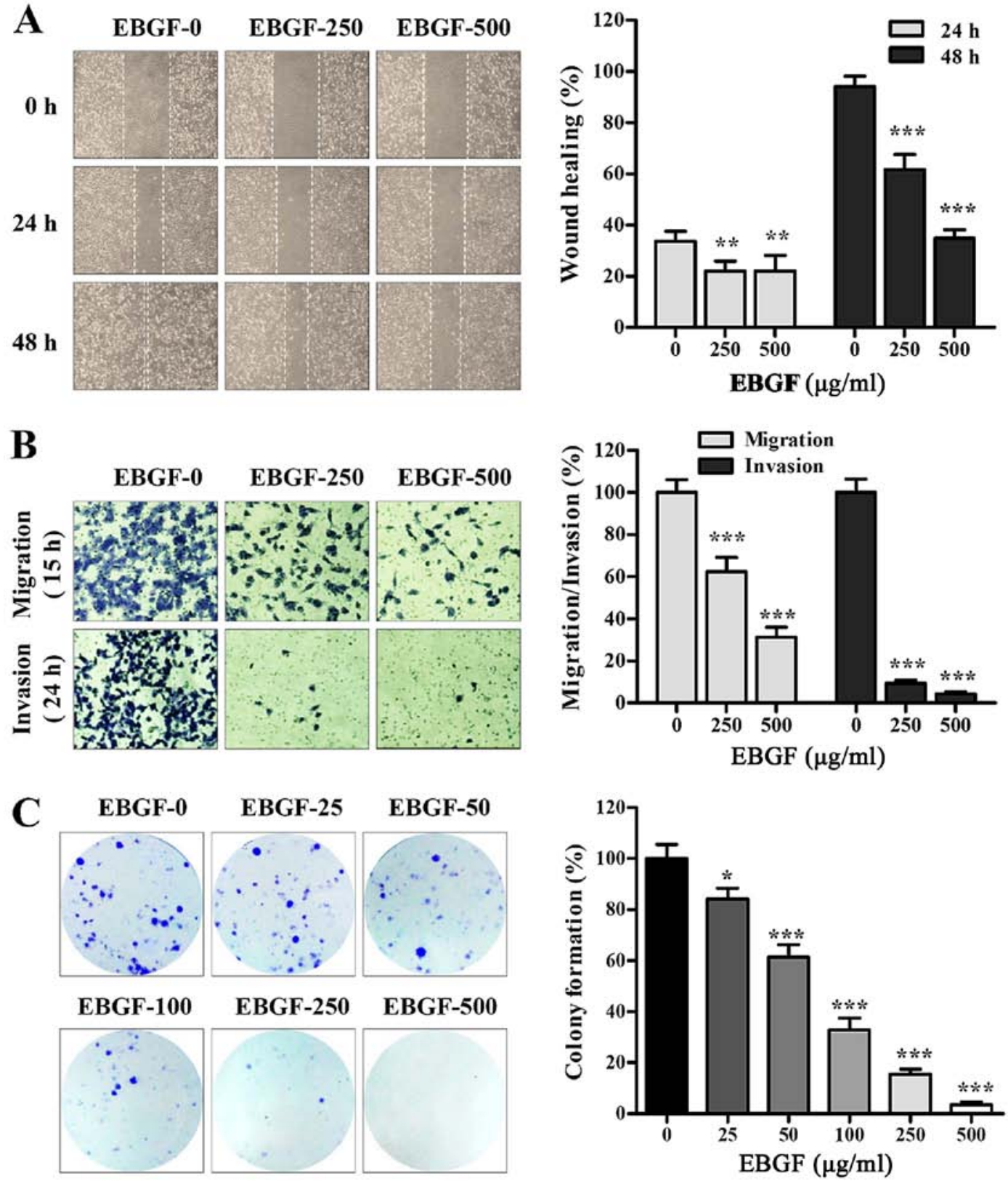

Figure 2. EBGF reduces metastatic activity of HT1080 cells. (A) Mitomycin C-treated monolayer cells were scratched, and the effects of EBGF on the wound healing were assessed during $48 \mathrm{~h}$. Wound width was measured in five selected fields, and relative wound healing was calculated on the basis of the width at $0 \mathrm{~h}$. (B) Transwell migration and invasion of EBGF-treated and untreated cells were examined after $15 \mathrm{hnd} 24 \mathrm{~h}$, respectively, and representative images of the lower surface are shown. The number of cells in five random fields was counted for each group, and relative migration and invasion were calculated. (C) The colony formation ability from a single cell was assessed in the presence or absence of EBGF. After incubation for 7 days, cells were stained with crystal violet solution, and sizable colonies were counted ( $\mathrm{n}=3$ per group). Data are representative of three independent experiments and are expressed as the mean $\pm \mathrm{SD}$. Statistical significance was determined using Student's t-test. ${ }^{*} \mathrm{p}<0.05,{ }^{* *} \mathrm{p}<0.01$, and ${ }^{* * * *} \mathrm{p}<0.001$ vs. untreated control.

respectively ( $\mathrm{F}=145.7, \mathrm{p}<0.0001$, one-way ANOVA) (Fig. 4C). These results collectively indicate that EBGF efficiently antagonizes tumor-induced angiogenesis.

EBGF decreases levels of angiogenesis-associated proteins under hypoxic conditions via suppression of the HIF-la pathway. Angiogenesis is essential for tumor growth and metastasis and depends on the release of pro-angiogenic factors by tumor cells and the surrounding cells. Because EBGF had inhibitory effects on tumor-induced angiogenesis, levels of pro-angiogenic factors in the control CM and EBGF-treated $\mathrm{CM}$ were analyzed using a Human Angiogenesis Proteome
Profiler array. As shown in Fig. 5A, EBGF treatment under hypoxic conditions significantly decreased the production of pro-angiogenic factors, including serpine E1, EGF, MMP-9, pentraxin, Ang-2, PDGF, uPA, IL-8, VEGF, and PIGF. Under hypoxic conditions, HIF-1 $\alpha$ is a key transcriptional regulator for the production of angiogenesis-related proteins. Consistent with the inhibitory effects on tumor-induced angiogenesis, EBGF strongly suppressed hypoxia-induced HIF-1 $\alpha$ accumulation and Akt/mTOR phosphorylation (Fig. 5B and 5C). In addition, cells with nuclear HIF-1 $\alpha$ under hypoxic conditions were significantly decreased by EBGF treatment in a dosedependent manner $(\mathrm{F}=492.2, \mathrm{p}<0.0001$, one-way ANOVA) 

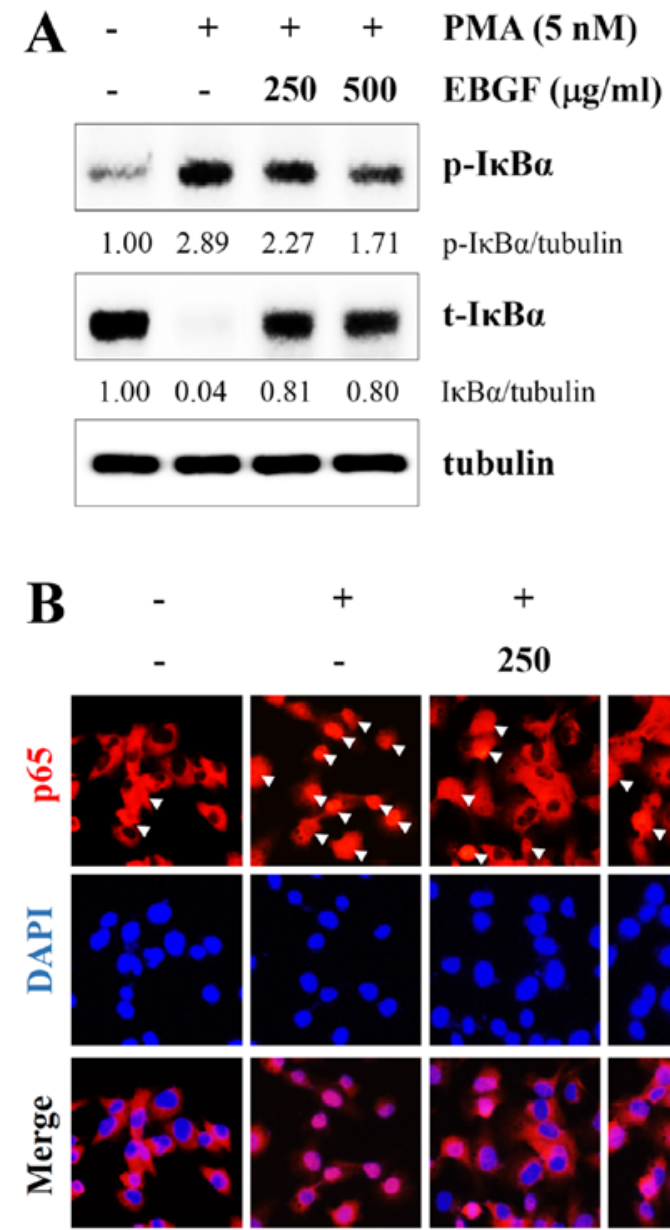

(a)

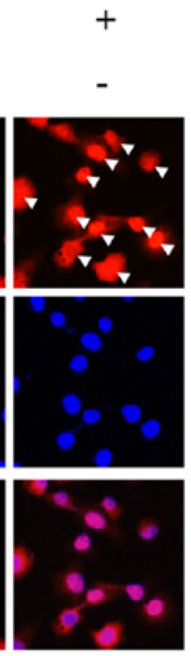

(b)

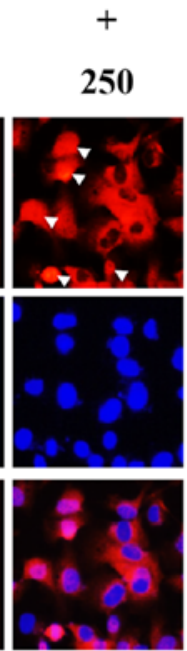

(c)

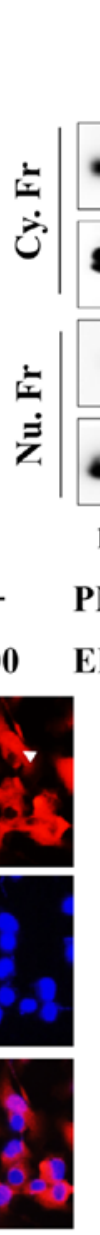

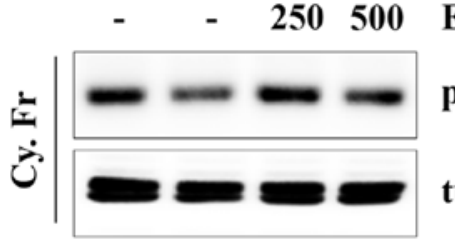

PMA (5 nM)

EBGF $(\mu \mathrm{g} / \mathrm{ml})$

p65

tubulin

p65

TBP

$\begin{array}{lllll}1.00 & 27.74 & 10.25 & 9.88 \quad \text { Nu-p65/Cy-p65 }\end{array}$

PMA (5 nM) (d)

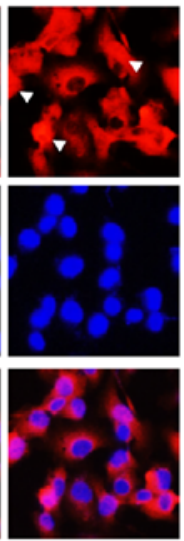

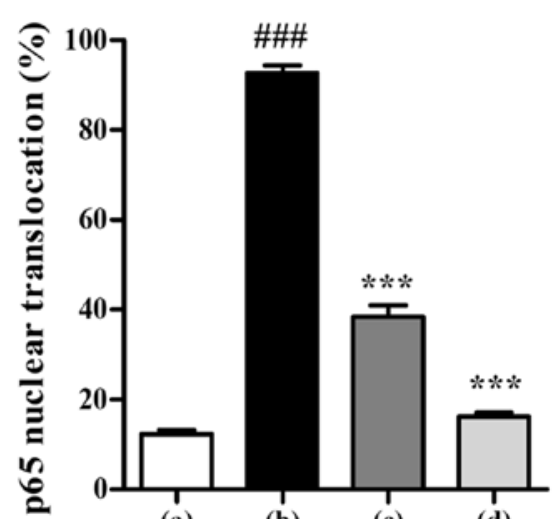

(a) (b) (c) (d)

Figure 3. EBGF blocks PMA-induced NF- $\kappa$ B activation in HT1080 cells. (A) Cells treated with 250 and $500 \mu \mathrm{g} / \mathrm{ml}$ EBGF for $12 \mathrm{~h}$ were stimulated with PMA

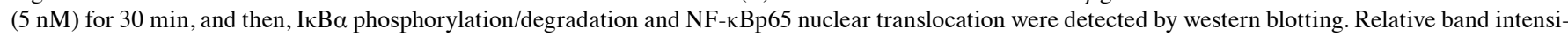
ties were calculated after normalization to tubulin and TBP expression as loading controls. Blots are representative of three independent experiments. (B) Cells in glass bottom dishes were treated with EBGF (250 and $500 \mu \mathrm{g} / \mathrm{ml})$ followed by stimulation with PMA (5 nM) for $30 \mathrm{~min}$. Using immunocytochemistry, nuclear translocation of NF- $\kappa$ Bp 65 was observed. DAPI was used to counterstain the nuclei, and white arrows indicate NF- $\mathrm{B}$ B 65 translocated to the nucleus. Data are shown as the mean \pm SD of five selected fields per sample and are representative of three independent experiments. Statistical significance was determined using Student's t-test. ${ }^{\# \#} \mathrm{p}<0.001$ vs. untreated control, ${ }^{* * *} \mathrm{p}<0.001$ vs. PMA control.

(Fig. 5D). These results indicate that EBGF reduced the production of angiogenesis-related proteins via suppression of the HIF-1 $\alpha$ pathway.

EBGF administration suppresses in vivo pulmonary metastasis of B16F10 cells with no side effects. To assess the anti-metastatic activity of EBGF in vivo, we examined the extent of pulmonary colonization of intravenously injected B16F10 cells in saline- and EBGF-treated C57BL/6J mice. In saline-treated control mice, B16F10 cells efficiently metastasized to the lungs and formed many colonies $(217.8 \pm 44.3)$, while EBGF administration at 50 and $100 \mathrm{mg} / \mathrm{kg}$ reduced the number of colonies to $122.2 \pm 16.4$ and $91.8 \pm 24.9$, respectively $(\mathrm{F}=22.78, \mathrm{p}<0.0001$, one-way ANOVA) (Fig. 6A). In addition, lung weight was also significantly decreased in EBGF-treated mice compared with that of control mice, which corresponded to the degree of pulmonary colonization of tumor cells, and resulted in an $\sim 60 \%$ reduction $(\mathrm{F}=49.20$, $\mathrm{p}<0.0001$, one-way ANOVA) (Fig. 6B). During the experiments, the differences in the body weight between control mice and EBGF-administered mice were insignificant (Fig. 6C). To further confirm the safety of EBGF in vivo, body weight and organ weight were measured after daily administration of EBGF at 50 and $100 \mathrm{mg} / \mathrm{kg}$ or saline to normal mice with no tumors for 21 days. As shown in Fig. 6D, EBGF-treated mice were not affected in terms of organ weight and body weight and showed similar levels compared with those of control mice, indicating that EBGF suppressed pulmonary metastasis of B16F10 cells without causing toxicity.

\section{Discussion}

Formation of new blood vessels in tumor masses is an important step in tumor progression and metastasis. Without angiogenesis, solid tumors cannot grow larger than a few millimeters and cannot form metastatic foci in distant organs; thus, targeting the vasculature of tumors is recognized as a promising strategy for cancer therapy $(26,27)$. Tumor cells can release positive regulators of angiogenesis, mobilize an angiogenic stimulator from ECMs, and recruit host cells that 

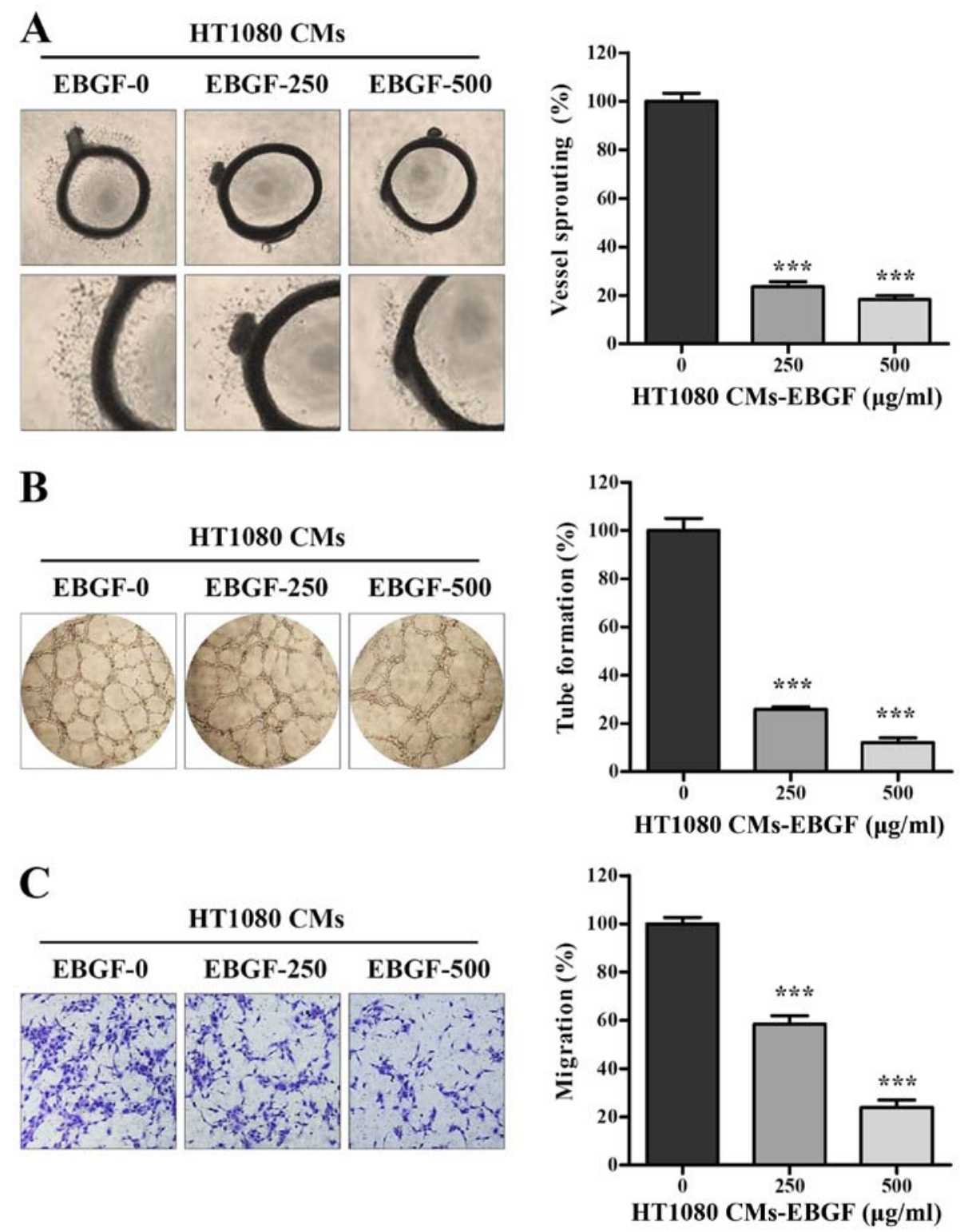

Figure 4. EBGF attenuates tumor-induced angiogenesis. (A) Rat aortic rings were placed on Matrigel-coated 48-well culture plates and were incubated in EGM-2 for 3 days. After removing EGM-2, aortic rings with microvessel sprouting were further incubated in EBGF-treated or untreated HT1080 CM for 5 days, and images were photographed. The degree of microvessel sprouting was measured using ImageJ software and is expressed as the mean \pm SD of three different samples. (B) HUVECs $\left(5 \times 10^{4}\right)$ suspended in EBGF-treated or untreated HT1080 CM (100 $\mu$ l) were incubated in BME-coated 96-well plates for 4-12 h. The ability of HUVECs to form capillary-like network was determined, and the number of tubes in three different samples was counted. (C) For the Transwell migration of HUVECs, EBGF-treated or untreated HT1080 CM $(650 \mu \mathrm{l})$ was added to the lower chamber, and HUVECs $\left(1 \times 10^{5} / 100 \mu \mathrm{l}\right)$ were added to the upper chamber of the Transwell and were then incubated for $15 \mathrm{~h}$. After staining of migrated HUVECs adhering to the lower surface with crystal violet solution, the number of cells in three random fields was counted and is expressed as the mean \pm SD. Experiments were repeated at least three times. Statistical significance was determined using Student's t-test. ${ }^{* * *} \mathrm{p}<0.001$ vs. control CM.

produce angiogenic proteins, leading to the enhancement of EC proliferation, migration, and cell-cell and cell-ECM adhesion. Among pro-angiogenic proteins, VEGF is overexpressed in most cancers and is closely associated with increased microvessel density of tumors and poor prognosis in cancer patients. In addition, VEGF is upregulated in tumor masses under hypoxic conditions via the HIF-1 $\alpha$ pathway and promotes transition from dormant avascularized tumors to outgrowing vascularized ones (28-31). Therefore, drugs targeting VEGF production and the VEGF-mediated signal transduction pathway have been developed and evaluated in a large number of clinical trials for cancer therapy. Neutralizing antibodies to VEGF, such as bevacizumab, have been approved by the
US FDA and used in combination with chemotherapy to treat metastatic colorectal cancer, non-small cell lung cancer, renal cell carcinoma, ovarian cancer, and others. Tyrosine kinase inhibitors targeting VEGFR, such as sunitinib and sorafenib, also have been approved for treating hepatocellular carcinoma, renal cell carcinoma, and others (27).

Despite their high efficacy, these anti-angiogenic treatments exhibited several limitations, including narrow adaptation, side effects, and resistance, and failed to induce a long-term response in the majority of patients. Thus, alternative approaches to provide better outcomes, fewer side effects, and more benefits are needed. Phytochemicals including curcumin, resveratrol, genistein, and sulforaphane 
A

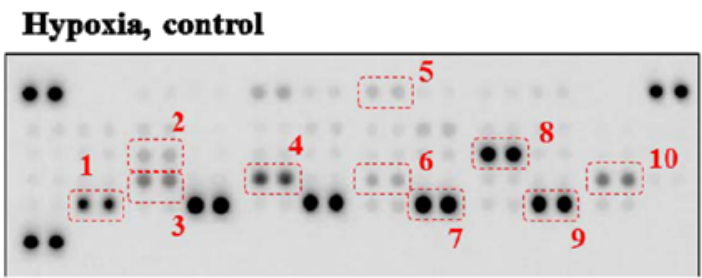

Hypoxia, EBGF $500 \mu \mathrm{g} / \mathrm{ml}$
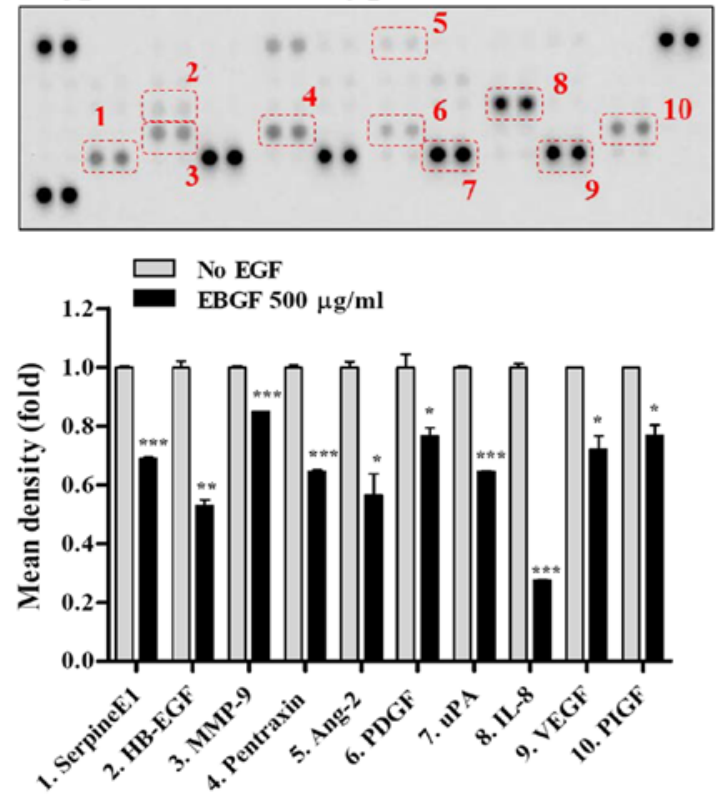

B

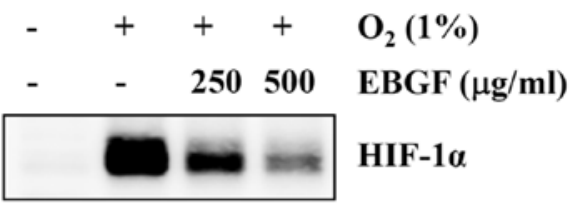

$\begin{array}{lllll}1.00 & 22.89 & 14.77 \quad 8.12 \quad \text { HIF-l } \alpha / \text { tubulin }\end{array}$

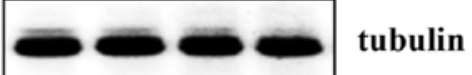

C
D

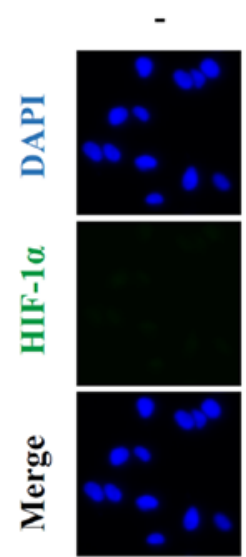

(a)

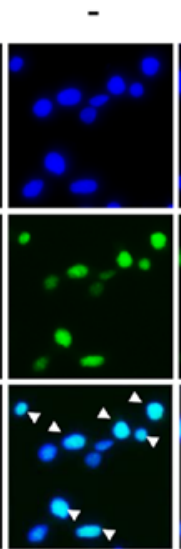

(b)

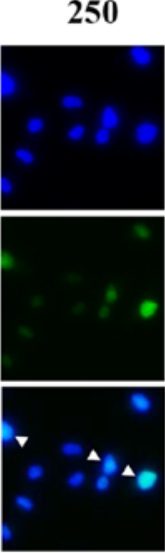

(c)

$+\mathrm{O}_{2}(1 \%)$

$500 \quad$ EBGF $(\mu \mathrm{g} / \mathrm{ml})$

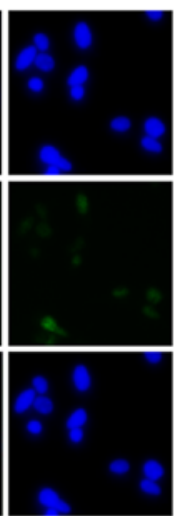

(d)

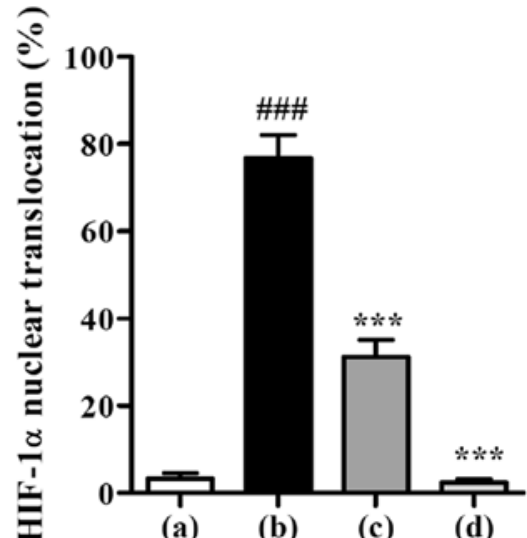

(a) (b) (c) (d)

Figure 5. EBGF inhibits the production of pro-angiogenic proteins and HIF-1 $\alpha$ nuclear expression in response to hypoxia. (A) Using a human angiogenesis array kit, levels of 55 angiogenesis-related proteins in CM obtained from EBGF-treated or untreated $\mathrm{HT} 1080$ cells under hypoxic conditions ( $1 \%$ O $\mathrm{O}_{2}$ ) were analyzed. Arrays are representative of two independent experiments, and relative pixel intensity was calculated using ImageJ software and is expressed as the mean \pm SD of duplicated dots. Statistical significance was determined using Student's t-test. " $\mathrm{p}<0.05,{ }^{* *} \mathrm{p}<0.01$, and ${ }^{* * *} \mathrm{p}<0.001 \mathrm{vs}$. control CM. (B and C) HT1080 cells were incubated in the presence or absence of EBGF and then exposed to hypoxia $\left(1 \% \mathrm{O}_{2}\right)$ for $6 \mathrm{~h}$. The levels of HIF-1 $\alpha$ and phosphorylated Akt and mTOR in total cell lysates were detected using western blotting. Relative band intensities were calculated after normalization to tubulin expression as the loading control. (D) Cells were incubated with EBGF (250 and $500 \mu \mathrm{g} / \mathrm{ml})$ in glass-bottom dishes for $12 \mathrm{~h}$ and then exposed to hypoxia (1\% $\mathrm{O}_{2}$ ) for $6 \mathrm{~h}$. Using immunocytochemistry, nuclear-translocated HIF-1 $\alpha$ was assessed. DAPI was used for counterstaining the nuclei, and white arrows indicate HIF-1 $\alpha$ translocated to the nucleus. Data are shown as the mean \pm SD of five selected fields per sample and are representative of three independent experiments. Statistical significance was determined using Student's t-test. ${ }^{\# \#} \mathrm{p}<0.001$ vs. normoxic control, ${ }^{* * * *} \mathrm{p}<0.001$ vs. hypoxic control.

have been shown to target numerous angiogenesis mediators and have anti-angiogenic effects in various cancers (32). In addition, herbal drugs, such as Cordyceps militaris, Patrinia scabiosaefolia, Ocimum gratissimum, Salvia officinalis, Eupatorium fortunei, and Anisi stellate fructus, have also been reported to have anti-angiogenic activities (22,33-36). 
A

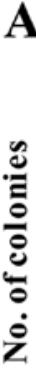
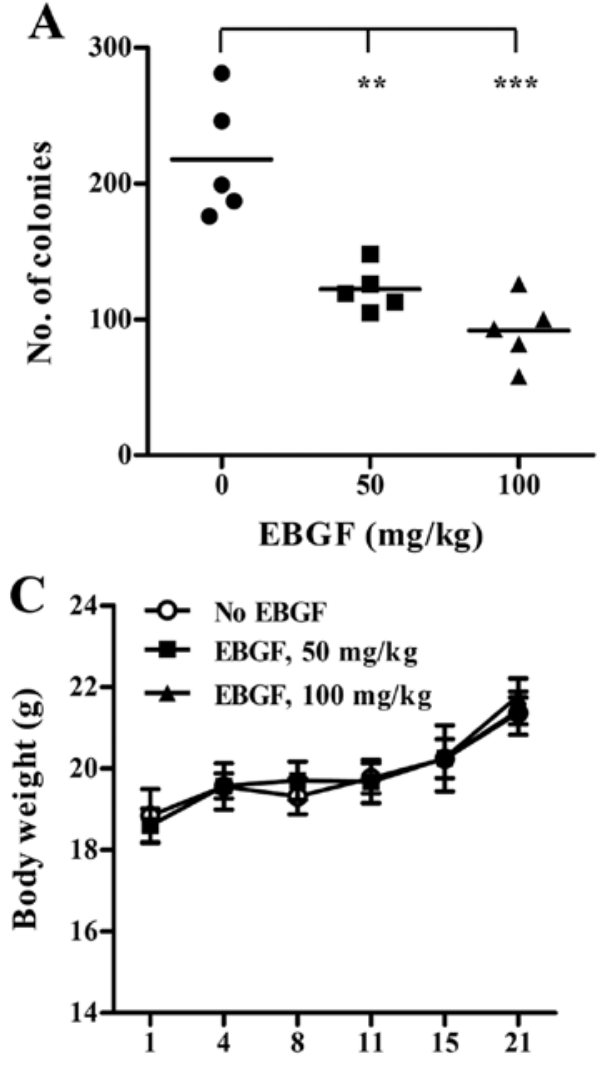

Days after tumor injection
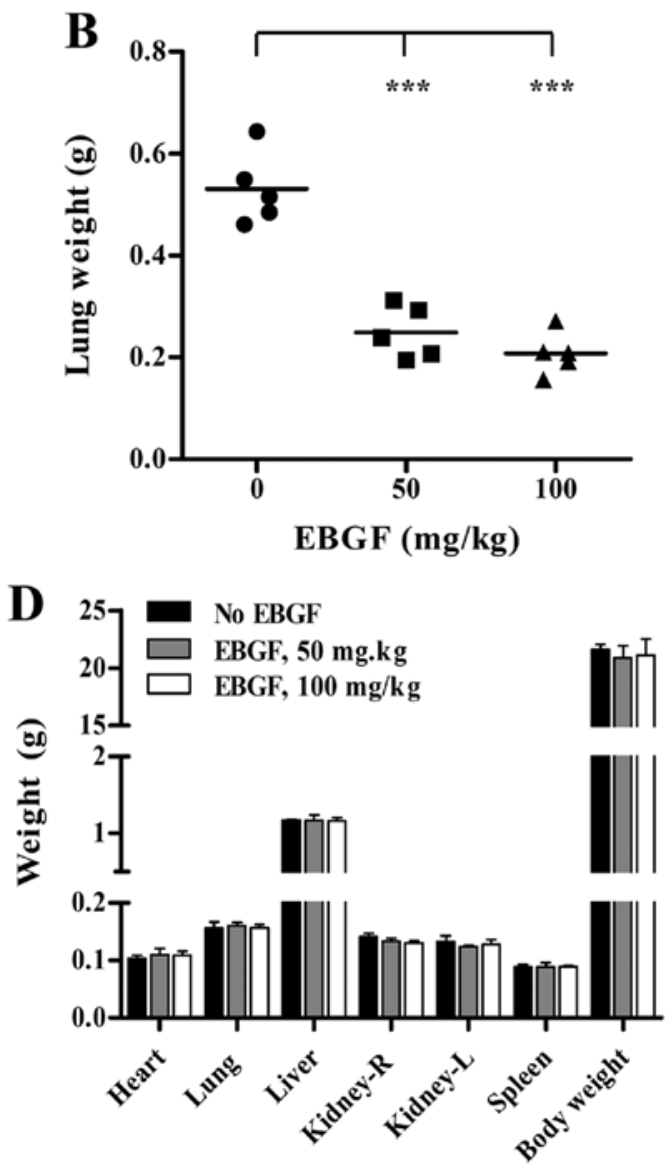

Figure 6. EBGF administration suppresses in vivo pulmonary metastasis of B16F10 cells with no adverse effects. (A and B) After intravenous injection of B16F10 cells (2x105/200 $\mu \mathrm{l}$ PBS/mouse), mice were randomly divided into 3 groups and orally administered saline or 50 and 100 mg/kg of EBGF daily for 21 days. On day 21 , metastasized colonies on the lung surface were counted, and the lungs were weighed. Bars represent the mean value of each group ( $\mathrm{n}=5$ per group). Statistical significance was determined using Student's t-test. ${ }^{* *} \mathrm{p}<0.01$ and ${ }^{* * *} \mathrm{p}<0.001$ vs. saline. (C) During the experiments, body weights were measured on days 1, 4,8,11, 15 and 21. (D) Each group of mice ( $\mathrm{n}=3$ per group) was orally administered saline, 50 and $100 \mathrm{mg} / \mathrm{kg}$ of EBGF daily for 21 days. After sacrifice, body weight and weight of organs were measured. Data are expressed as the mean \pm SD.

Interestingly, administration of Cordyceps militaris or ethanol extract of Patrinia scabiosaefolia (EEPS) efficiently reduced tumor volumes in colorectal cancer or melanoma via suppression of tumor angiogenesis, strongly supporting the role of angiogenesis in tumor progression $(33,34)$.

In a previous study, crocetin, a major component of GF, strongly suppressed TPA-induced skin carcinogenesis via reduction of TPA-induced nuclear proto-oncogenes, such as c-jun, c-fos, and c-myc, in mouse epidermis (37). In addition, stir-baked GF extract inhibited the branching structure of HT1080 cells in a three-dimensional (3-D) culture and suppressed MMP-16 activity against DQ-gelatin (21), but it did not affect cell viability. Geniposide and genipin are known to be major components of GF and are responsible for various pharmacological effects, including hepatoprotective, neuroprotective, anti-oxidative, antiinflammatory, and anti-thrombotic activities. Genipin induced apoptosis in HepG3 hepatocarcinoma cells, PC-3 prostate cancer cells, and HeLa cervical cancer cells (38). In addition, genipin reduced MMP-2 activity via upregulation of TIMP-1 and p38 activation, leading to suppression of the metastatic potential of human hepatocarcinoma cells at non-toxic doses (39). Ethanol extracts of GF and its n-butanol fraction were previously reported to possess potent anti-angiogenic activity in the chick embryo chorioallantonic membrane (CAM) assay
(40). Geniposide had potent anti-angiogenic activity and inhibited the growth of transformed NIH3T3 cells (41).

In this study, we demonstrated that EBGF at non-toxic doses dramatically reduced the PMA-induced increase in the expression of MMP-9, MMP-13, and UPA in HT1080 cells via suppression of NF- $\kappa \mathrm{B}$ activation (Figs. 1 and 3); consequently, wound migration, colony formation, and migration/invasion through Matrigel in a Transwell system were relatively lower in EBGF-treated cells than those in untreated control cells (Fig. 2). In addition, we observed that HT1080 control CM promoted sprouting of microvessels from aortic rings and capillary-like tube formation and migration of HUVECs, while EBGF-treated HT1080 CM weakly induced these angiogenic changes, indicating that EBGF suppressed the production of pro-angiogenic factors from HT1080 cells (Fig. 4). Under hypoxic conditions, HT1080 cells released pro-angiogenic factors, including serpine E1, EGF, MMP-9, pentraxin, Ang-2, PDGF, uPA, IL-8, VEGF, and PIGF, which stimulate microvascular EC proliferation, enhance EC migration and sprouting, inhibit EC apoptosis, and increase EC permeability. In contrast, EBGF-treated HT1080 cells produced these pro-angiogenic factors to a lesser extent compared with those of control HT1080 cells via suppression of the HIF-1 $1 \alpha / \mathrm{Akt} / \mathrm{mTOR}$ pathway, supporting the anti-angiogenic potential of EBGF (Fig. 5). We also observed that daily 
oral administration of EBGF effectively suppressed pulmonary metastasis of B16F10 melanoma cells in C57BL/6 mice, and no adverse reactions, including loss of body weight and changes in organ weight, were observed (Fig. 6). In conclusion, this study demonstrates the anti-metastatic and anti-angiogenic activities of EBGF in detail, and our data suggest that EBGF can be used as a safe and potent herbal medicine for treating patients with metastatic malignant tumors.

\section{Acknowledgements}

This study has been supported by the Grant K16281 awarded to Korea Institute of Oriental Medicine (KIOM) from Ministry of Science, ICT and Future Planning (MSIP), Republic of Korea.

\section{References}

1. Kumar S and Weaver VM: Mechanics, malignancy, and metastasis: The force journey of a tumor cell. Cancer Metastasis Rev 28: 113-127, 2009.

2. Hanahan D and Weinberg RA: The hallmarks of cancer. Cell 100: 57-70, 2000.

3. Weidner N, Semple JP, Welch WR and Folkman J: Tumor angiogenesis and metastasis - correlation in invasive breast carcinoma. N Engl J Med 324: 1-8, 1991.

4. Stamenkovic I: Matrix metalloproteinases in tumor invasion and metastasis. Semin Cancer Biol 10: 415-433, 2000.

5. Stetler-Stevenson WG and Yu AE: Proteases in invasion: Matrix metalloproteinases. Semin Cancer Biol 11: 143-152, 2001.

6. John A and Tuszynski G: The role of matrix metalloproteinases in tumor angiogenesis and tumor metastasis. Pathol Oncol Res 7: $14-23,2001$.

7. Weidner N, Carroll PR, Flax J, Blumenfeld W and Folkman J: Tumor angiogenesis correlates with metastasis in invasive prostate carcinoma. Am J Pathol 143: 401-409, 1993.

8. Stetler-Stevenson WG: Matrix metalloproteinases in angiogenesis: A moving target for therapeutic intervention. J Clin Invest 103: 1237-1241, 1999.

9. Gupta MK and Qin RY: Mechanism and its regulation of tumorinduced angiogenesis. World J Gastroenterol 9: 1144-1155, 2003.

10. Nussenbaum F and Herman IM: Tumor angiogenesis: Insights and innovations. J Oncol 2010: 132641, 2010.

11. Papetti M and Herman IM: Mechanisms of normal and tumorderived angiogenesis. Am J Physiol Cell Physiol 282: C947-C970, 2002.

12. Baeriswyl V and Christofori G: The angiogenic switch in carcinogenesis. Semin Cancer Biol 19: 329-337, 2009.

13. Hida K, Kawamoto T, Ohga N, Akiyama K, Hida Y and Shindoh M: Altered angiogenesis in the tumor microenvironment. Pathol Int 61: 630-637, 2011.

14. Aburada M, Sasaki H and Harada M: Pharmacological studies of Gardeniae fructus. II. Contribution of the constituent crude drugs to choleretic activity of 'Inchinko-to' in rats (author's transl). Yakugaku Zasshi 96: 147-153, 1976 (In Japanese).

15. Miyasita S: A historical study of Chinese drugs for the treatment of Jaundice. Am J Chin Med Gard City N Y 4: 239-243, 1976.

16. Koo HJ, Song YS, Kim HJ, Lee YH, Hong SM, Kim SJ, Kim BC, Jin C, Lim CJ and Park EH: Antiinflammatory effects of genipin, an active principle of gardenia. Eur J Pharmacol 495: 201-208, 2004.

17. Lin YJ, Lai CC, Lai CH, Sue SC, Lin CW, Hung CH, Lin TH, Hsu WY, Huang SM, Hung YL, et al: Inhibition of enterovirus 71 infections and viral IRES activity by Fructus gardeniae and geniposide. Eur J Med Chem 62: 206-213, 2013.

18. Toriizuka K, Kamiki H, Ohmura NY, Fujii M, Hori Y, Fukumura M, Hirai Y, Isoda S, Nemoto Y and Ida Y: Anxiolytic effect of Gardeniae Fructus-extract containing active ingredient from Kamishoyosan (KSS), a Japanese traditional Kampo medicine. Life Sci 77: 3010-3020, 2005.

19. $\mathrm{Hu} \mathrm{QH}$, Zhu JX, Ji J, Wei LL, Miao MX and Ji H: Fructus Gardenia extract ameliorates oxonate-induced hyperuricemia with renal dysfunction in mice by regulating organic ion transporters and mOIT3. Molecules 18: 8976-8993, 2013.
20. Zhang HY, Liu H, Yang M and Wei SF: Antithrombotic activities of aqueous extract from Gardenia jasminoides and its main constituent. Pharm Biol 51: 221-225, 2013.

21. Yang JG, Shen YH, Hong Y, Jin FH, Zhao SH, Wang MC, Shi XJ and Fang XX: Stir-baked Fructus gardeniae (L.) extracts inhibit matrix metalloproteinases and alter cell morphology. J Ethnopharmacol 117: 285-289, 2008.

22. Kim A, Im M and Ma JY: Anisi stellati fructus extract attenuates the in vitro and in vivo metastatic and angiogenic potential of malignant cancer cells by downregulating proteolytic activity and pro-angiogenic factors. Int J Oncol 45: 1937-1948, 2014.

23. Kim A, Im M, Yim NH, Kim T and Ma JY: A novel herbal medicine, KIOM-C, induces autophagic and apoptotic cell death mediated by activation of JNK and reactive oxygen species in HT1080 human fibrosarcoma cells. PLoS One 9: e98703, 2014.

24. Kim A, Im M, Gu MJ and Ma JY: Citrus unshiu peel extract alleviates cancer-induced weight loss in mice bearing CT-26 adenocarcinoma. Sci Rep 6: 24214, 2016.

25. Kim A, Im M, Yim NH and Ma JY: Reduction of metastatic and angiogenic potency of malignant cancer by Eupatorium fortunei via suppression of MMP-9 activity and VEGF production. Sci Rep 4: 6994, 2014.

26. Albini A, Tosetti F, Li VW, Noonan DM and Li WW: Cancer prevention by targeting angiogenesis. Nat Rev Clin Oncol 9: 498-509, 2012

27. Jayson GC, Kerbel R, Ellis LM and Harris AL: Antiangiogenic therapy in oncology: Current status and future directions. Lancet 388: 518-529, 2016.

28. Abdelrahim M, Konduri S, Basha R, Philip PA and Baker $\mathrm{CH}$ : Angiogenesis: An update and potential drug approaches (Review). Int J Oncol 36: 5-18, 2010.

29. Lin SC, Liao WL, Lee JC and Tsai SJ: Hypoxia-regulated gene network in drug resistance and cancer progression. Exp Biol Med (Maywood) 239: 779-792, 2014.

30. Liao D, Corle C, Seagroves TN and Johnson RS: Hypoxiainducible factor-1alpha is a key regulator of metastasis in a transgenic model of cancer initiation and progression. Cancer Res 67: 563-572, 2007.

31. Zhou J, Schmid T, Schnitzer S and Brüne B: Tumor hypoxia and cancer progression. Cancer Lett 237: 10-21, 2006.

32. Wang Z, Dabrosin C, Yin X, Fuster MM, Arreola A, Rathmell WK, Generali D, Nagaraju GP, El-Rayes B, Ribatti D, et al: Broad targeting of angiogenesis for cancer prevention and therapy. Semin Cancer Biol 35 (Suppl): S224-S243, 2015.

33. Chen L, Liu L, Ye L, Shen A, Chen Y, Sferra TJ and Peng J: Patrinia scabiosaefolia inhibits colorectal cancer growth through suppression of tumor angiogenesis. Oncol Rep 30: 1439-1443, 2013.

34. Ruma IM, Putranto EW, Kondo E, Watanabe R, Saito K, Inoue Y, Yamamoto K, Nakata S, Kaihata M, Murata H, et al: Extract of Cordyceps militaris inhibits angiogenesis and suppresses tumor growth of human malignant melanoma cells. Int J Oncol 45: 209-218, 2014.

35. Nangia-Makker P, Tait L, Shekhar MP, Palomino E, Hogan V, Piechocki MP, Funasaka T and Raz A: Inhibition of breast tumor growth and angiogenesis by a medicinal herb: Ocimum gratissimum. Int J Cancer 121: 884-894, 2007.

36. Keshavarz M, Mostafaie A, Mansouri K, Bidmeshkipour A, Motlagh HR and Parvaneh S: In vitro and ex vivo antiangiogenic activity of Salvia officinalis. Phytother Res 24: 1526-1531, 2010.

37. Hsu JD, Chou FP, Lee MJ, Chiang HC, Lin YL, Shiow SJ and Wang CJ: Suppression of the TPA-induced expression of nuclearprotooncogenes in mouse epidermis by crocetin via antioxidant activity. Anticancer Res 19B: 4221-4227, 1999.

38. Cao H, Feng Q, Xu W, Li X, Kang Z, Ren Y and Du L: Genipin induced apoptosis associated with activation of the c-Jun NH2-terminal kinase and p53 protein in HeLa cells. Biol Pharm Bull 33: 1343-1348, 2010.

39. Wang N, Zhu M, Tsao SW, Man K, Zhang Z and Feng Y: Up-regulation of TIMP-1 by genipin inhibits MMP-2 activities and suppresses the metastatic potential of human hepatocellular carcinoma. PLoS One 7: e46318, 2012.

40. Park EH, Joo MH, Kim SH and Lim CJ: Antiangiogenic activity of Gardenia jasminoides fruit. Phytother Res 17: 961-962, 2003.

41. Koo HJ, Lee S, Shin KH, Kim BC, Lim CJ and Park EH: Geniposide, an anti-angiogenic compound from the fruits of Gardenia jasminoides. Planta Med 70: 467-469, 2004. 